Inheritance and motivation in Construction Morphology

\title{
Geert Booij
}

Leiden University Centre of Linguistics

P.O. Box 9515

2300 RA Leiden

The Netherlands

February, 2014

[Draft, not for quotation or copying]

To appear as a chapter of

Nikolas Gisborne and Andrew Hippisley (eds.), Defaults in morphological theory. Oxford: Oxford University Press. 
Inheritance and motivation in Construction Morphology

\section{Geert Booij}

\section{Introduction}

The basic question to be addressed in this chapter is: what is the status of the notions 'inheritance' and 'default inheritance' in the theoretical framework of Construction Morphology (CM)? This framework, developed in Booij (2010), assumes a hierarchical lexicon with both abstract morphological schemas and stored complex words that instantiate these schemas. Consider the English agent noun eater, derived from the verb eat by means of suffixation with -er. The relevant word formation schema that underlies the formation of this noun can be represented as follows:

$$
<\left[[\mathrm{x}]_{\mathrm{V}} \text { er }\right]_{\mathrm{Nj}} \leftrightarrow\left[\text { Agent of } \mathrm{SEM}_{\mathrm{i}}\right]_{\mathrm{j}}>
$$

This constructional schema specifies that there is a systematic relation between nouns of the form [V-er] and the meaning 'Agent of the action expressed by the verbal base' (angled brackets delimit a constructional schema, the double-sided arrow denotes the correlation between form and meaning). The word eater is an instantiation of this word formation schema, coined by the unification of this schema with the lexical entry for the verb to eat. The word formation schema (1) has tow roles: it specifies how new agent nouns can be created, and it motivates the meaning of existing nouns of this type.

The lexicon of a language can be modeled in such a way that the abstract word formation schemas dominate their individual instantiations. Thus, the lexicon is partially conceived of as a hierarchical network in which lower nodes, the existing complex words, can be assumed to inherit information from dominating higher nodes. Thus, there is a 'vertical' relationship between the word formation schema and the complex word.

Information concerning a derived word is also related to that of its base word. The meaning of eater is partially a reflex of the meaning of to eat.

"A second way in which information about a complex word can be inherited is that the information concerning its base word is also part of the information concerning the 
complex word. That is, in addition to inheritance of information in a lexical entry from the schemas that dominate it, there is also inheritance of information from the base word. The word skyper coined through unification of the verb skype with the schema for deverbal nouns in -er [schema 1 in this chapter, GEB], inherits the information concerning skype. [...]

The relation between base word and derived word can be specified through co-indexation: the lexical index of the PHON[ological], SYN[tactic], and SEM[antic] properties of the base word appears as part of the PHON, SYN, and SEM information concerning the derived word as well.” (Booij 2010: 26-27)

This implies that inheritance is not only a relationship between an abstract schema and a complex word, but also between a complex word and its base. The first type of relation is that of instantiation, the second one is the subpart relation. This implies that the information concerning a complex word may be inherited from more than one source, that is, there is multiple inheritance. In the case of compounding, a compound will be linked to a compound schema, and to the subparts of the compound, which usually form independent lexical entries. For instance, the form and meaning of the compound pasta eater can be predicted from the form and meaning specified in the word formation schema for NN compounds, and those of the nouns pasta and eater.

What is the formal role of inheritance in the CM approach to word formation? Since, as we will see below, CM assumes for good reasons that lexical items are fully specified, CM does not use inheritance in order to achieve underspecification of lexical entries, with their predictable properties omitted. Therefore, the notion 'inheritance' has to receive a different interpretation, that of 'motivation': a word formation schema motivates the existence of an individual complex word to the extent that it predicts some or all of its properties.

The notion of default inheritance, which will be discussed in detail in section 4, therefore has to receive a new interpretation as well. The empirical question is: which kind of properties specified in word formation schemas can be overridden for an individual complex word without obliterating the motivation relation? The same question can be asked about the relationship between a complex word and its base, since not all properties of a base word may recur in the corresponding derived word.

These issues are discussed step by step in the following sections. Section 2 discusses the notion of inheritance in an impoverished entry theory, and section 3 argues in favour of a full entry theory. In sections 4 and 5 I argue that default inheritance can be reinterpreted in CM as partial motivation, and that it is important to determine which properties of a word formation 
schema are defeasible. In section 6 the various types of motivation of form-meaning relationships in the lexicon are briefly discussed, and section 7 shows how systematic polysemy may be present in word formation schemas and subschemas. In section 8 I argue that allomorphy need not block the motivating role of a base word, and that full phonological compositionality can therefore be overridden. Section 9 summarizes my findings.

2. Inheritance in an impoverished entry theory.

In the debate on the architecture of grammar, ideas about the nature and functions of the lexicon play a central role. A key issue in this debate is the balance between storage and computation. In one strand of linguistic theorizing, going back to Bloomfield (1935), the lexicon is the basic list of irregularities. That is, whatever can be computed by rule should not be stored in the lexicon. This idea applies to both morphological and syntactic constructions. This idea has been defended for morphology most eloquently by Steven Pinker in his book Words and Rules, in which he argues that only irregular past tense forms of English verbs are stored, whereas regular past tense forms are not stored but computed on the fly (Pinker 1999).

There is a great deal of evidence, however, that regular words can be stored in the lexicon (Baayen et al. 2003; Bybee 2006; De Vaan et al. 2007). This raises the question of how exactly words, whether regular or irregular, are specified in the lexicon. Will they be fully specified, or only their unpredictable properties?

One possible answer to this question is given by the 'impoverished entry theory'. In this theory, words with shared properties are dominated by nodes in a hierarchical lexicon on which these common properties are specified, and these properties are then inherited from the dominating schema. In the case of complex words, these dominating nodes are the abstract word formation schemas. Specification of these properties can therefore be omitted from the individual lexical entries. This idea is developed in various forms in (Deo 2007; Flickinger 1987;

Riehemann 1998; Sag et al. 2003). The 'impoverished entry theory' thus belongs to the tradition in which one tries to achieve maximal parsimonious lexical representations for individual words (Deo 2007).

What about fully regular complex words? In the Pinker (1999) approach these words will lack an entry in the lexicon, as they are fully predictable. However, this approach does not account for the conventional aspects of the lexicon. For instance, suppose that the complex word doable is completely regular in terms of its form and semantics. The lexical description of this deverbal adjective, derived from the transitive verb $d o$, is something like: 


$$
\left[[\text { do }]_{\mathrm{Vi}} \text { able }\right]_{\mathrm{Aj}} \leftrightarrow\left[\text { can undergo action } \mathrm{SEM}_{\mathrm{i}}\right]_{\mathrm{j}}
$$

This is the format of a lexical entry in grammars with parallel architecture (Jackendoff 2002). On the left we find the phonological specification of the morphological components of the word and its morphosyntactic structure, and on the right hand side the semantics of the word is specified. By means of co-indexation, the contribution of the morphological constituents to the meaning of the word as a whole is specified.

Pace Pinker (1999), we want to specify that this adjective exists, i.e. it belongs to the lexical convention of English speakers (who know that doable is a word they have come across before). If we want to omit all predictable information about doable, this adjective has to be represented as follows:

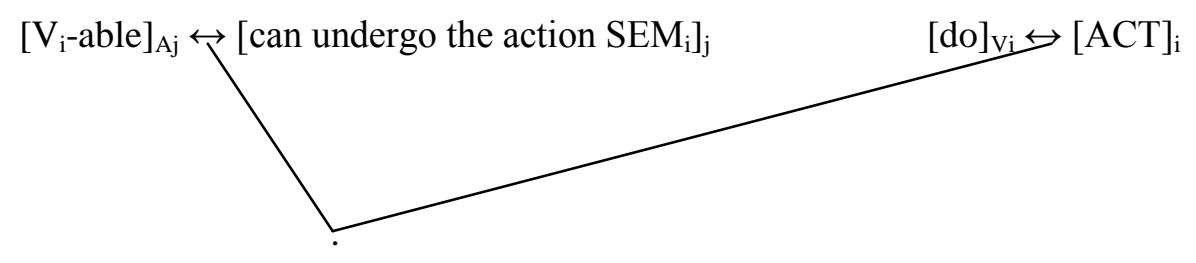

The dot indicates that the adjective doable exists, but that all its formal and semantic properties are inherited from the morphological schema to which it is linked, and from its base word, the verb $d o$ with the meaning of the semantic primitive ACT, with which it is co-indexed.

This representation of a fully regular individual complex word by means of a dot, linked to the relevant morphological schema and to its base word, is a consequence of the impoverished entry theory. All that remains to be specified is the fact that the possible word doable actually exists. This is a parsimonious approach to the lexicon, in which all redundancy is avoided.

The difference between this approach and the words-and-rules approach of Pinker (1999) is that the presence of fully regular complex words in the lexicon is accounted for, as illustrated in (3). We have to specify which complex words belong to the conventional lexicon, even if they are fully regular. For example, Noun to Verb conversion in English is a commonly used word formation process, but language users know which verbal conversions are OK (compare the established verb to saddle with the non-existent verb to mustard) since this process is semiproductive (Jackendoff 1997). The alternative would be that we specify for each noun whether the schema of Noun to Verb conversion applies to it. 
The same observation can be made for lexical rules that do not change word class but do create new lexical subentries. For instance, the English verb sleep can be used intransitively, but also transitively, as in This hotel sleeps 100 guests. This lexical rule cannot be applied, however, to the verb eat, since a sentence like This restaurant eats 100 guests is unavailable for the intended interpretation that this is a restaurant that can provide food for 100 guests.

In sum, it is clear that even in an impoverished entry theory we have to list the individual words that have been derived by means of some lexical rule. However, this still leaves open whether we should omit predictable properties of words. This issue is discussed in the next section.

\section{Arguments for a full entry theory}

There are a number of problems for the impoverished entry theory. The general objection to this approach is that it does not do justice to the insight that our lexical memory is vast, and that there is therefore no good reason to adopt a parsimonious approach to the lexicon. This objection is related to a general criterion for adequacy in linguistic models. A linguistic model must allow for "graceful integration" (Jackendoff 2011), that is, it must be in harmony with the findings of other linguistic subdisciplines such as psycholinguistics, language acquisition theory, and historical linguistics, and with those of cognitive science in general.

Consider what we know about morphological language acquisition. Morphological schemas are acquired on the basis of a set of memorized complex words, i.e. fully specified complex words. For instance, the user of English first acquires individual deverbal nouns in -er, such as baker and writer, and after sufficient exposure to a set of such words, the schema for deverbal nouns in -er will be grasped. It would be odd to assume that once speakers have discovered the schema, they will delete the predictable information concerning individual complex words that they have already stored in their lexical memory (Jackendoff 1997). "Once a generalization has been made on the basis of stored instances, those instances may be redundant but there is no mechanism for deleting them from memory, so we must assume that at least these stored cases persist" (Hudson 2007: 22). There is no psychological pressure to delete predictable information, once acquired, given the vastness of human memory. In terms of processing it is also advantageous that one does not need to compute properties of memorized complex words before using them, as they can be retrieved directly from the mental lexicon which will speed up processing. 
Thus, the acquisition of morphological knowledge appears to be parallel to that of syntactic knowledge, as found in various studies by Tomasello (Tomasello 2003): the acquisition of syntactic patterns starts with the acquisition of specific verbs together with their syntactic frames, and gradually knowledge of more abstract syntactic patterns emerges.

This relationship between the knowledge of individual complex words and that of abstract patterns can also be concluded from the fact that there is a positive correlation between growth of one's individual lexicon and acquisition of morphological knowledge. For instance, Smedts (1979) investigated the word formation competence of 13-year-old Flemish Dutchspeaking children, and concluded that this competence is still restricted at that age compared to that of adult language users, and that this correlates with not yet fully developed knowledge of the lexical norm (the set of conventional, established complex words) of one's native language. The only word formation process that these children were able to use properly and productively was compounding, the most productive and frequently used word formation process of Dutch (Smedts 1979). This shows that language acquisition in this domain is relatively slow, and depends to a great extent on the growth of one's mental lexicon.

The full entry model is a basic ingredient of the model of the hierarchical lexicon in CM, as developed in Booij (2010), which can be characterized as follows:

(i) Constructional schemas specify the predictable information of classes of fully specified existing complex lexical items, and specify how similar new complex words can be coined. (ii) Constructional schemas may dominate subschemas that specify additional or more specific properties of subclasses of lexical items.

This model does justice to the fact that knowledge and use of abstract morphological schemas is dependent upon the memorized knowledge of sets of complex words that instantiate these patterns.

Therefore, morphological schemas should not be seen as formal mechanisms for achieving maximally parsimonious lexical representations. Instead, they have two other functions: schemas motivate the existence of the relevant set of complex words, and they predict how this set can be extended (cf. Goldberg 2006 for the same point for syntactic constructions). The motivating function of schemas has the effect of reducing the degree of arbitrariness of formmeaning relations in complex words in the lexicon. Schemas also structure the lexicon. For instance, the schema for English deverbal nouns in -er in (1) defines a word family with shared properties, and thus provides a partial structure to the English lexicon.

The full entry theory is also defended in Jackendoff (1975; 1997). Jackendoff (1997) concluded that given a full entry theory, schemas have to play a role in computing the 
informational cost attached to a lexical item. This is an old idea, first introduced in Jackendoff (1975).

"What seems to be lost in the full entry theory, though, is any notion that semiregularities "save" anything: shelve takes no fewer bits to list than an underived word of similar complexity. In order to make sense of the full entry theory, then, we need to develop an alternative notion of what constitutes "informational cost" in the lexicon." (Jackendoff 1997: 129).

In this chapter, I will elaborate a theory which makes use of both schemas and full entries in terms of the notion of 'motivation'. Morphological schemas, besides having the function of specifying how new complex words can be formed, reduce the memory burden of the lexicon, as they reduce the degree of arbitrariness in the set of correspondences between form and meaning of words specified in the lexicon.

A second argument in favour of a full entry theory is given by Zeschel (2009), and may be characterized as the indeterminacy argument. Zeschel's point is that it is not always easy, given a vast amount of naturalistic data, to determine exactly the set of common properties of a set of related expressions. Moreover, as speakers may differ in the amount of information they have accumulated, they may also differ in the kind of generalizations that they can make:

"As a result, certain properties of the investigated pattern will have the character of statistical rather than strictly mandatory features. However, adopting the criterion of (non-)predictability forces the analyst to be fully explicit about precisely which features are required for inclusion and exactly where (i.e. on which level of schematicity) they are encoded, which [...] may be quite difficult to determine" (Zeschel 2009: 187-88).

In a full-entry model we can account for the properties of individual expressions (syntactic or morphological constructions), and have flexibility in determining and stating the common properties of sets of constructions. Therefore, given the choice of a full entry model, we have to reconsider the role of the notions 'inheritance' and 'default inheritance'. This is the topic of the next two sections.

\section{Default inheritance}


The concept of default inheritance is linked primarily to the impoverished entry theory. Yet, as we will see below, it can also be used as a concept in the full entry model, and it is therefore important to discuss its status in more detail.

The basic idea of default inheritance as used in a theory of the hierarchical lexicon is the following: a word inherits its properties from a dominating node (schema or constraint) unless specified otherwise. "Default inheritance [...] is useful in the statement of subregularities and idiosyncratic exceptions, without sacrificing morphological generalizations" (Deo 2007). Bouma (1993: 50-52) also gives a series of arguments for the use of default inheritance in syntactic and morphological descriptions.

Here is the definition of 'default inheritance' (in an impoverished entry framework):

"Default inheritance.

If $\mathrm{X}$ and $\mathrm{Y}$ are nodes, $\mathrm{X}$ may inherit from $\mathrm{Y}$ if a fact identifying $\mathrm{Y}$ as an inheritance source is included at X. All attribute: value pairs at $Y$ become available at X, except those having an attribute which is already present in an attribute: value pair at X." (Corbett \& Fraser 1993: 116)

As this definition makes clear, the basic presupposition of this approach is that all information about the properties of a word is given in the form of attribute: value (also called feature: value) pairs. If a property can be overridden by a contrary specification on the level of the individual word, this property (feature-value combination) is marked as defeasible by means of a slash (/) (Sag et al. 2003). Properties may be absolute, hence indefeasible.

The use of default inheritance in the feature-based framework of Categorial Unification Grammar has been argued for by Bouma (1993). The basic idea is that local information may override inherited information (default unification). Default unification is an operation which unifies parts of one feature structure (the default argument) with another feature structure (the non-default argument). The non-default argument must always be preserved, that is, this type of unification is only monotonic with respect to the non-default argument.

A morphological example that may be used to illustrate this idea involves nominal compounds in Dutch. These are right-headed, so the gender of the compound (neuter or nonneuter) is determined by the head noun. However, there are a few compounds for which this does not hold. In the compound spleet-oog 'slit-eye (offensive)', which denotes human beings, the head noun has the feature [neuter] for gender, and thus it is predicted that this compound should be neuter as well. However, this compound has [non-neuter] gender for semantic reasons. This 
must then be specified in the lexical entry for this noun, and this non-default feature value will override the inherited feature value [neuter] which follows from the general word formation schema for Dutch nominal compounds. ${ }^{1}$

Not all linguists who use an impoverished entry model want to employ default inheritance, as it is a very powerful device, and may lead to arbitrary classifications.

"Information in non-monotonic hierarchies can be overwritten arbitrarily often, so there is an unlimited number of possibilities for representing data in such hierarchies." "The most important decision in constructing a non-monotonic hierarchy is about what information should be regarded as regular." (Kilbury et al. 2006: 430)

The degree of arbitrary overwriting can be reduced by distinguishing between absolute and defeasible properties. Riehemann (1998), in her analysis of German deverbal adjectives in -bar '-able', rejects default inheritance, and shows how exceptions can be accounted for in a monotonic lexical hierarchy without default inheritance. Her argument against default inheritance is based on language acquisition: "if all information were defeasible it would be unclear how the schemata would be formed" (Riehemann 1998: 72). However, she points out that by making a distinction between absolute and defeasible properties one "may be able to deal with the acquisition problem and avoid wrong classifications of new items to be entered in the hierarchy" (Riehemann 1998: 72).

Indeed, lexical hierarchies with default inheritance can be rephrased as lexical hierarchies without default inheritance, by setting up a more complicated hierarchy of subcategories, as shown for German adjectives in -bar in Riehemann (1998). This has also been pointed out by Müller (2007).

One instance where we might use default inheritance is in the description of the class of Dutch deverbal adjectives in -baar '-able'. In the regular case, the base word is a transitive verb (4a). However, there are various classes of exceptions as to the category of the base word:
type of base word
examples
a. V-transitive
eet-baar 'edible', drink-baar 'drink-able'
b. V-ergative
vloei-baar 'liquid', brand-baar 'inflammable'
c. V-intransitive
leef-baar 'livable', werk-baar 'work-able'
d. $\mathrm{N}$
vrucht-baar 'fruit-bearing, fruitful'
e. not existing
dier-baar 'dear-baar, dear' 
The adjectives in (4b-c) do not have transitive verbs as bases, the adjective vruchtbaar in (4d) has a noun as its base, and the category of the base in dier-baar (4e) is indeterminable as dier is a cranberry morpheme. In the deverbal adjectives (4b-c), the default value for the attribute '(sub)category of the base word', namely 'transitive verb', is overridden. We may therefore conclude that the subcategory of the base verb is a defeasible property.

The words in $(4 \mathrm{~d}, \mathrm{e})$ are even more exceptional as the base is not a verb at all. Does this mean that 'syntactic category of the base' is a completely defeasible property? This depends on whether it makes sense to relate the latter two words to the general schema for deverbal adjectives. That is, in a default inheritance approach we have to decide which properties are defeasible. If we decide that the lexical category $\mathrm{V}$ of the base word is an absolute property, these two words cannot be linked to the general word formation schema for -baar adjectives. The only regularity is that we can be sure of their status as adjectives on the basis of the presence of -baar. Their meaning is completely different from that of the deverbal -baar adjectives as they do not express the meaning components of 'possibility' and 'passive'. Therefore, we might consider specifying the verbal nature of the base word as an absolute property of -baar adjectives, as this will block the possibility of relating the adjectives vruchtbaar and dierbaar to the schema for deverbal adjectives in -baar. However, we then miss the generalization that these words are adjectives. Below we will see that there are cases where input category should be a defeasible category. Therefore, I assume that the input category of a word formation schema can be overridden by an individual complex word. However, in this situation, the complex word will have a lower degree of motivation.

As absolute properties of this class of words one may count that they are adjectives and that the suffix is -baar, not e.g. -(e)lijk, although we find adjectives with the same meaning ending in -(e)lijk, e.g. drag-elijk 'bear-able'. Hence, I assume that the only absolute, nondefeasible properties of word formation schemas are 'output category' and 'phonological form of the affix' (apart from allomorphy).

As these examples show, there is no automatic procedure to determine which properties are absolute. The choice of absolute properties should depend on the limits of motivation. Since the adjective drag-elijk 'bearable' cannot be seen as motivated by the word formation schema for deverbal adjectives ending in -baar, this adjective should not be related to it. It must receive its motivation from another schema.

The various kinds of Dutch adjectives in -baar can be modelled in the following nonmonotonic hierarchy with default override: 


$$
\left[\mathrm{V}_{\mathrm{tr}} \text {-baar }\right]_{\mathrm{A}}
$$

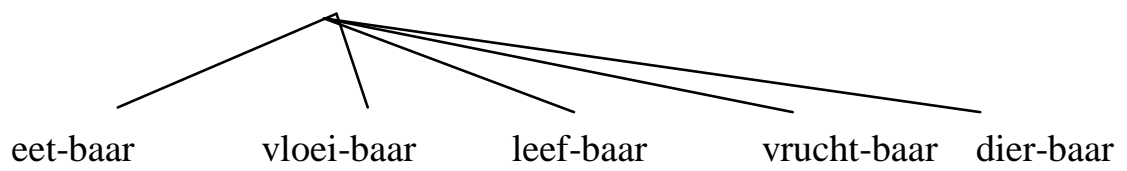

In this tree, only eet-baar has the default properties, while the other adjectives, all listed in (4), have properties that override the predicted category of the base word.

As mentioned above, Riehemann (1998) opted for a monotonic lexical hierarchy, without default inheritance. The Dutch facts mentioned above can be modelled monotonically, without default inheritance, in the following hierarchy:

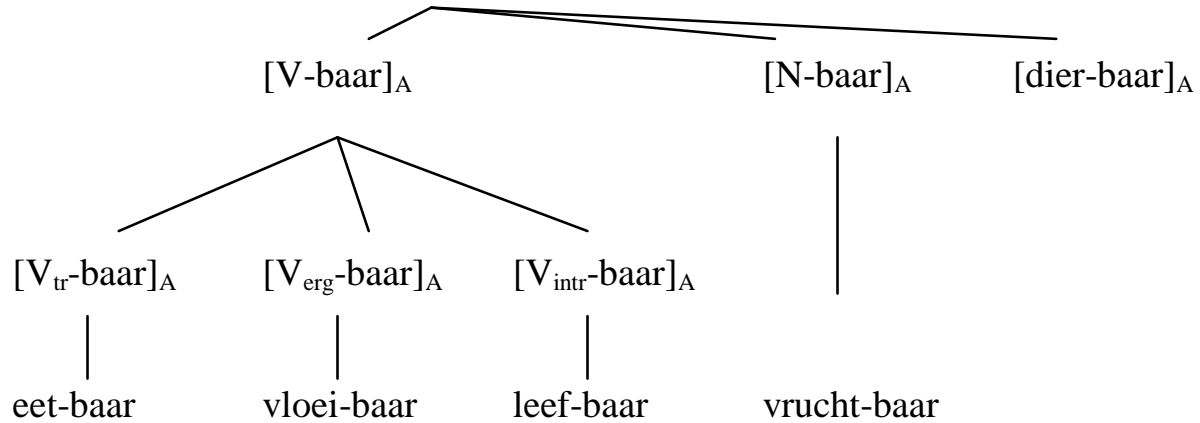

What this hierarchy does not express, however, is that only one of the patterns is productive, namely the leftmost subschema on the bottom line. Hence, in this approach we have to assign labels such as 'productive' or 'regular' to specific subschemas if we want to express this difference. This is indeed what Riehemann (1998: 64) does in a similar schema for German -bar adjectives, which contains a subschema with transitive base verbs with the label 'regular'. The alternative is to assume only one schema, with transitive verbs as bases, and to consider the other classes as cases of default override. This predicts that the formation of new German adjectives in -bar will only be productive for base words which are transitive verbs, a correct prediction.

A challenge for default inheritance in combination with an impoverished entry-theory is how to deal with idiosyncratic semantic properties of derived words, since it is not so obvious how to specify all sorts of semantic properties by means of attribute: value combinations. For instance, the Dutch adjective drink-baar 'drinkable' not only has the expected literal meaning, but also the meaning 'pleasant to drink'. Similarly, the adjective betaal-baar, derived from the verb betaal 'to pay', means not only 'payable' but also 'affordable'. In these cases, the special 
meaning can be seen as an (optional) additional semantic property, specified in the lexical representation of these adjectives, which does not clash with the set of inherited properties. But what to do if the idiosyncratic meaning overrides the literal meaning? For instance, if speakers of Dutch call wine ondrinkbaar 'undrinkable', the literal meaning 'not drinkable' is superseded, as this adjective does not mean that the wine cannot be drunk, but that it is not pleasant to drink it. So the meaning of a complex word as specified by a word formation schema has to be defeasible, at least in principle, if we want to link the word ondrinkbaar to the relevant morphological schema. This raises the question whether semantic properties specified by word formation schemas are always defeasible, and also whether semantic idiosyncrasies can always be specified in terms of contrary attribute: value specifications. The latter issue depends on the kind of semantic metalanguage one wishes to use. Since this issue is far beyond the goals of this paper, I will not dwell on it at this point. What is relevant here is that in a full-entry theory of word formation, the precise way in which one formalizes the notion 'degree of motivation of a complex word' can be kept open.

To sum up, what we have seen so far is that the notion of 'default inheritance' has the advantage of enabling us to specify the regular properties of a set of complex words, while at the same time allowing for specification of exceptional properties of individual words without necessitating a complex hierarchy of classes of exceptions, as would be necessary in a theory of inheritance without default override. However, making use of default override raises the question of how to make a principled distinction between absolute and defeasible properties, and how to model the override of semantic properties.

As concerns semantic properties, one may wonder if it is always possible to describe them in terms of attribute: value combinations. The semantic properties of individual complex words that are not predicted by the relevant morphological schema often result from the universal mechanisms of polysemy: metonymy and metaphor. It is hard to model these forms of semantic default override in terms of defeasible attribute: value combinations.

In the next section, I will discuss the status of default inheritance in a full-entry theory such as Construction Morphology, and argue that in this model we can accommodate the basic insight behind the concept of default inheritance.

\section{Inheritance as motivation}

As argued above, and as in Booij (2010), Construction Morphology requires a full-entry model of lexical representation. Therefore, the formal issues concerning default inheritance discussed in 
the previous section do not arise in the same way. The relation between a schema and its dependents, the individual lexical items, is one of motivation. Motivation means that there is a non-arbitrary relationship between the form and meaning of a linguistic construct. This is why Goldberg (2006: 215) opts for the concept of default inheritance, even if it is technically speaking not true that lexical items are underspecified and inherit information from dominating nodes in a hierarchical lexicon. Complex words are in principle motivated by the schema by which they are dominated. Newly derived complex words get their predictable properties through the unification of word formation schemas and base words, but these predictable properties are not omitted in their lexical representations. Constructional schemas can have this motivation function, because they always deal with the relationship between form and meaning. There may be various degrees of motivation, as the individual complex words may have deviating properties. That is, the interpretation of the concept of default inheritance in a full-entry model of lexical knowledge is that a complex word may have properties that are not motivated by the morphological schema by which it is dominated, and of which it is an instantiation.

Note, however, that the basic question raised by the idea of default override remains the same: which properties of a schema are absolute, and can never be overridden? In other words: what are the constraints on linking a complex word to a motivating morphological schema? In the words of Luger \& Stubblefield (1993: 388), quoted by Hudson (2007: 25) "If we define a penguin as a bird that does not fly, what is to prevent us from asserting that a block of wood is a bird that does not fly, does not have feathers, and does not lay eggs?".

The usual defining properties of a word formation process are: the syntactic category of input and output, the phonological shape of the affix, and the semantic correlate of the morphological construction. For instance, the following schema may be assumed for Dutch adjectives ending in -baar:

$$
\left.<\left[[\mathrm{x}]_{\mathrm{Vi}} \text { baar }\right]_{\mathrm{Aj}} \leftrightarrow\left[\text { property of y[y can undergo action } \mathrm{SEM}_{\mathrm{i}}\right]\right]_{\mathrm{j}}>
$$

Of these properties, phonological shape is an obvious candidate for being an absolute property. For example, it does not make any sense to consider an adjective in -elijk such as drag-elijk 'bearable' as being motivated by schema (6), even though this word has the relevant meaning, since the form of a word is always the starting point for determining its motivation.

What about input and output category? The output category of a word formation schema and its phonological form should be seen an absolute properties, since these are the defining properties of morphological schemas. For instance, it does not make sense to relate English 
adjectives ending in -ish to the word formation schema for English adjectives in -able, or adjectives in $-y$ such as blood-y to a schema for diminutive nouns in $-y$ like Andy $(<$ Andrew).

On the other hand, the input category may have the status of defeasible property. This can be illustrated as follows: in the set of Dutch deverbal adjectives in -baar there are a few words in which the verbal base is not transitive. These are incidental violations, as it is not the case that these classes of adjectives can be extended systematically. Speakers of Dutch are able to recognize these words as being motivated by schema (5) since the semantic components of 'possibility' and 'undergoing an event' are still present. This is in line with the view defended by Plag that the output category and the semantic properties of a morphological process are crucial for its definition (Plag 2004). Therefore, we can consider these adjectives as being motivated by schema (6). This implies that we must consider as defeasible the constraint that the input verb must be transitive.

Let us now consider the variation in input category in Dutch diminutive nouns. Although these diminutives are normally derived from nouns, they are also occasionally derived from base words of other categories, as shown in (7):

(7) Dutch diminutives

category base diminutive

$\begin{array}{lll}\text { N } & \text { vrouw 'woman' } & \begin{array}{l}\text { vrouw-tje 'little woman, sweetheart' } \\ \text { A }\end{array} \\ \text { V } & \text { lief 'sweet' } & \begin{array}{l}\text { lief-je 'sweetheart' } \\ \text { dut-je 'to nap' }\end{array} \\ \text { Num } & \text { tien 'ten' } & \text { tien-tje '10 guilder note' } \\ \text { Adv } & \text { uit 'out' } & \text { uit-je 'outing' } \\ \text { NP } & \text { twaalf uur '12 o'clock' } & \text { twaalfuur-tje 'packed lunch' } \\ \text { PP } & \text { onder ons 'between us' } & \text { onderons-je 'private chat' } \\ \text { Pronoun } & \text { dit en dat 'this and that' } & \text { dit-je-s en dat-je-s 'odds and ends' }\end{array}$

These data show that violations of the input constraint $\mathrm{N}$ do exist. These deviations are characteristic of a very productive category that may expand its domain of application occasionally to base words of other categories. Hence, we may consider these diminutive nouns as being motivated by the word formation schema for denominal diminutive nouns:

$$
<\left[[\mathrm{x}]_{\mathrm{Ni}}(\mathrm{t}) \mathrm{je}\right]_{\mathrm{Nj}} \leftrightarrow[\text { SMALL SEM }]_{\mathrm{j}}>
$$


There are no systematic violations of the input category constraint, since it is not possible to coin new diminutive nouns from base words other than nouns in a systematic fashion. By linking the diminutive nouns with base words other than nouns to schema (8) we claim that they are partially motivated by this schema. That complex words may be only partially motivated is the consequence of these words having properties that override the properties predicted by the dominating word formation schema.

Let us now look at a case of Dutch suffixation, in which the suffix takes base words of various categories:

$$
\begin{array}{lll}
\text { base } & \text { stem } & \text { derived adjective } \\
\mathrm{N} & \text { rots 'rock' } & \text { rots-achtig 'rocky' } \\
\mathrm{V} & \text { weiger 'to refuse' } & \text { weiger-achtig 'refusing persistently' } \\
\mathrm{A} & \text { groen 'green' } & \text { groen-achtig 'greenish' }
\end{array}
$$

These examples show that the suffix -achtig is promiscuous, and takes Ns, Vs and As as base words. When combined with an $\mathrm{N}$, the suffix -achtig has three systematic meanings: 'like N', 'possessing N', and 'liking N':

$$
\begin{aligned}
& \text { soldaat-achtig 'soldier-like' } \\
& \text { rots-achtig 'rock-possessing, rockys' } \\
& \text { pasta-achtig 'pasta-liking, pasta-minded' }
\end{aligned}
$$

Given this array of facts, one may wonder whether we can characterize the word formation process with the suffix -achtig as being constrained to nouns, with occasional override of this constraint by deverbal and deadjectival adjectives in -achtig. The answer is negative, because we would miss the generalization that attachment of -achtig to verbs and adjectives is productive and systematic as well. Moreover, there is also a systematic correlation between the category of the base word and the meaning contribution of the suffix. Hence, we have to specify the various subpatterns of adjective formation in -achtig, including the systematic polysemy for the subset of denominal adjectives. At the same time we want to express the property common to these word formation processes, namely the fact that they all create adjectives. We therefore need a lexical hierarchy of the following type $(\mathrm{R}=$ with some semantic relation to $)$ :

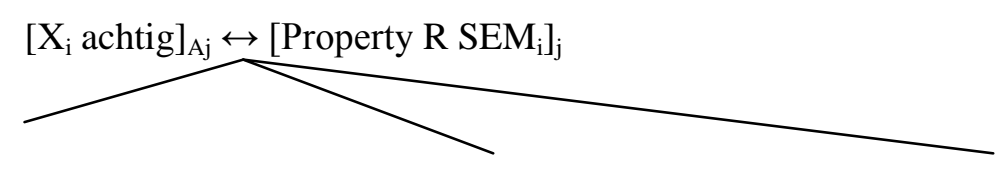




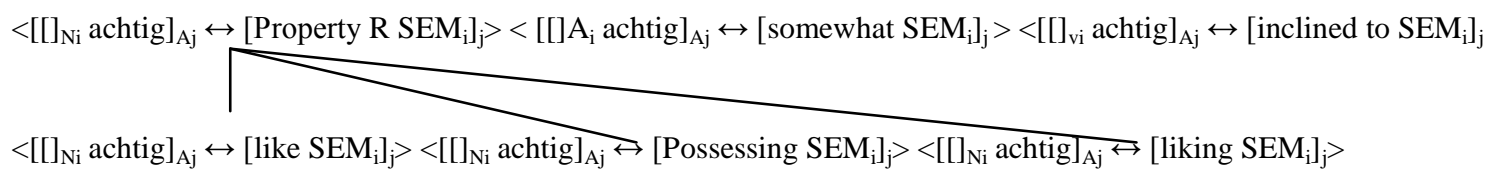

Thus, we see how a hierarchy with schemas and subschemas can do justice to the existence of systematic subclasses. The subschemas provide more detailed spell-out of properties mentioned by the dominating schema, and hence this is not a case of default override. If a complex word can be linked to a more specific subschema of word formation, this preserves the full motivation of that complex word, whereas in the case of default override for an individual complex word that word will lose some of its motivation.

This approach is a response to Plag's (2004) radical proposal that word formation processes are only constrained semantically, without the category of the base word playing a role. It appears that quite often there is a systematic correlation between the category of the base word and the semantics of the complex word that can be expressed in subschemas.

This approach implies that when faced with a word formation pattern with various subpatterns we have to decide between two descriptive options: one schema with default override, or one general schema with more specific subschemas. The basic criterion for making a choice is the productivity of each subpattern: if it is productive, there is good reason to assume a subschema. However, the problem for a consistent use of this criterion is that productivity is not a simple all-or-none phenomenon, and that productivity is a matter of degree. That is, there may be cases where the choice between the two options is not straightforward.

Complex words may thus differ in their degree of motivation, as not all properties may be predictable. This is confirmed by psycholinguistic research. For instance, it has been established in an experimental task that the word settlement is felt as more affixed than government, presumably because the meaning of settlement is more transparent, and directly derivable from the verb settle and the meaning contribution of the suffix -ment (Hay \& Baayen 2005). The word government denotes not only the act of governing but also the institution that governs. It is this latter meaning that is dominant, and therefore this word is less motivated than settlement.

Differences in degree of motivation of complex words are not only determined by the relation between a complex word and its dominating schema, the relation of instantiation. A complex word instantiates a morphological schema, and a morphological schema motivates a complex word. These relations are established by the formal operation of unification: a complex word arises through the unification of a (base) word and a morphological schema. However, the 
same operation of unification also establishes another type of lexical relation, that between a complex word and its base word. The meaning of a complex word is partially motivated by the meaning of its base word (or base words, in the case of compounding). That is, the notion of inheritance is also relevant for the relation between base word and complex word, since a complex word may be said to inherit part of its meaning from its base word.

As to the issue of degree of motivation, there is an interesting parallelism to be observed here: the properties of a complex word may not be fully motivated by the morphological schema that it is dominated by, as we saw above, but a complex word may also not be completely compositional as far as the meaning contribution of its base word(s) is concerned. For instance, the Dutch denominal adjective wonder-lijk 'strange', derived from the noun wonder 'miracle', has a meaning component 'strange' that is not present in wonder 'miracle'. The meaning of the adjective does not fully reflect the meaning of its base noun, and hence its degree of motivation is low. These observations suggest that we need a gradient notion of motivation of complex words. This is in line with Hay \& Bayen (2005), a survey of experimental research on awareness of morphological structure. They come to "the conclusion that morphological structure is indeed inherently graded" (Hay \& Baayen 2005: 346).

The interpretation of the relation of a word formation schema and its instantiations as motivation also throws light on a related issue: should we assume constructional schemas for word formation processes that are no longer productive? For instance, Dutch features a number of deverbal adjectives ending in -(e)lijk that are semantically similar to the Dutch -baar adjectives and the English -able adjectives, for instance:

$$
\begin{array}{ll}
\text { aanbid 'to adore' } & \text { aanbidd-elijk 'adorable' } \\
\text { bemin 'to love' } & \text { beminn-elijk 'lovable' } \\
\text { opmerk 'to note' } & \text { opmerk-elijk 'notable' } \\
\text { toegeef 'to indulge' } & \text { toegeef-lijk 'indulgent' }
\end{array}
$$

The meaning of these adjectives is motivated both by the meaning of their base verb and the meaning contribution of the suffix 'can be V-ed'. Hence, these adjectives are semantically motivated even though the underlying word formation process is no longer productive. This implies that we should allow for unproductive word formation schemas, and hence, word formation schemas must be labeled as productive or unproductive.

\section{Types of motivation}


As we saw above, a word formation schema reduces arbitrariness in the form-meaning correspondence of complex words, as does the relation between a complex word and its base word(s). Apart from these two types of motivating link for a complex word (instantiation link, subpart link) there are other types of motivation that will be briefly discussed here: paradigmatic relationships between complex words, and patterns of polysemy.

\subsection{Paradigmatic relations}

The role of paradigmatic relations in word formation has been discussed in Booij (2010: 31-36), partially on the basis of the following English word pairs for which there is no corresponding base word. For instance, there is no word altru or aut in English:

$\begin{array}{ll}\text { altru-ism } & \text { altru-ist } \\ \text { aut-ism } & \text { aut-ist } \\ \text { bapt-ism } & \text { bapt-ist } \\ \text { commun-ism } & \text { commun-ist } \\ \text { pacif-ism } & \text { pacif-ist }\end{array}$

Here is the relevant comment on these word pairs in (Booij 2010: 33):

"Even though they have no corresponding base word, the meaning of one member of a pair can be defined in term of that of the other member. In particular, the meaning of the word in -ist can often be paraphrased as 'person with the ability, disposition, or ideology denoted by the word in ism'. Hence, the following paradigmatic relationship can be defined for these two schemas:

$\left.<[\mathrm{x}-\mathrm{ism}]_{\mathrm{Ni}} \leftrightarrow \mathrm{SEM}_{\mathrm{i}}\right\rangle \approx\left\langle[\mathrm{x}-\mathrm{ist}]_{\mathrm{Nj}} \leftrightarrow\left[\text { person with property } \mathrm{Y} \text { related to } \mathrm{SEM}_{\mathrm{i}}\right]_{\mathrm{j}}>\right.$

where $\mathrm{SEM}_{\mathrm{i}}$ represents the meaning of the word in -ism. Thus, an altruist has a disposition for altruism, and a pacifist adheres to the ideology of pacifism. [..] The meaning of these nouns in -ist is not simply a compositional function of their constituent parts, but contains the meaning of a related word with the same degree of complexity." 
(The symbol $\approx$ indicates a paradigmatic relationship between schemas.)

The schema in this quotation is a second order schema, as it specifies the relation between two different schemas and hence two classes of complex words for which there is no subpart relation from the formal point of view. In other words, second order schemas also have a motivation function, and contribute to reducing the degree of arbitrariness between form and meaning of complex words.

\subsection{Polysemy}

First order and second order morphological schemas are not the only type of rules that reduce arbitrariness. There are also 'rules' of polysemy that motivate the co-existence of more than one meaning for a simplex or complex word.

A useful classification of polysemy has been provided by Blank, who distinguishes four subtypes (Blank 2003):

(i) rule-based, nonlexicalized polysemy (e.g. ham sandwich 'customer who has ordered a ham sandwich');

(ii) rule-based and lexicalized polysemy with no or few idiosyncratic restrictions (e.g. book 'material object, content');

(iii) rule-based and lexicalized polysemy with idiosyncratic restrictions (eg. school 'building, institution');

(iv) idiosyncratic lexicalized polysemy (e.g. mouse 'rodent animal, computer device').

It is obvious that polysemy of type (i) should not lead to new lexical subentries: this type of semantic extension is of a universal pragmatic nature. The implication of polysemy of types (ii)-(iv) is different, however, in that for each sub-meaning of a word, a separate lexical entry must be created.

In the case of type (iv) polysemy, as in mouse, the meaning 'computer device' can be understood as a metaphorical extension of the primary sense of mouse, but as it is obviously something one has to learn for this individual word, (at least) two lexical entries are required for mouse. In some cases different sub-meanings correlate with different syntactic properties. For instance, the Dutch noun voetbal 'football' can be used to denote 'the world of football'. This is a metonymic sense extension. In this interpretation it is an abstract noun, and this correlates with the neuter gender taken by the noun, whereas in the concrete interpretation 'type of ball' this 
noun is non-neuter. Thus, de voetbal denotes the ball with which to play, whereas het voetbal denotes the game of football, and the organizational structure for this type of game.

What about rule-based polysemy of the types (ii) and (iii)? Recall that the polysemy of ham sandwich is an effect of a universal pragmatic rule (based on metonymy) that one can refer to a person by mentioning an object related to that person. Hence, this does not require a separate lexical entry for the person interpretation of ham sandwich.

Polysemy may be based on lexical rules or schemas of sense extension ('regular polysemy'), which are motivated by the general mechanisms of metaphor and metonymy (that is, these lexical rules or schemas are instantiations of these mechanisms). The outputs of these rules have to be stored. They are similar to rules of conversion (usually subsumed under morphology) in that they create new lexical entries without effecting a change in phonological form.

When the polysemy of a certain word instantiates a more general pattern and is thus a case of regular polysemy, this does not imply that this polysemy does not have to be codified for each individual word of the relevant type. In this respect, 'lexical rules' of the type discussed here are similar to word formation rules, which also specify systematic, rule-governed relations between two lexical entries, a base word and its derivative. Both word formation schemas and lexical rules motivate properties of lexical items.

An example of a lexical pattern of polysemy that can be expressed by a lexical schema is the regularity that nouns that denote an institution can also be used to denote the building in which the institution operates, as well as the people attached to the institution. For instance, the word university may denote an educational institution, its building, and its employees.

(14) a. Harvard is an excellent university

b. The university burned down last night

c. The university will be on vacation next week

Hence, we may assume lexical schemas of the following type:

$$
\begin{array}{ll}
\text { a. } & <\mathrm{N}_{\mathrm{i}} \leftrightarrow \text { INSTITUTION }_{\mathrm{i}}>\approx<\mathrm{N}_{\mathrm{i}} \leftrightarrow \text { BUILDING of } \text { SEM }_{\mathrm{i}}> \\
\text { b. } & <\mathrm{N}_{\mathrm{i}} \leftrightarrow \text { INSTITUTION }_{\mathrm{i}}>\approx \mathrm{N} \leftrightarrow \text { PEOPLE related to } \text { SEM }_{\mathrm{i}}>
\end{array}
$$

Semantic extension patterns also apply to specific classes of complex words, that is, in the case of polysemous derivational affixes. A well known and much discussed case of polysemy in Germanic and Romance languages is that of suffixes with agentive meanings, which are also 
used to express instrumental and other meanings (Luschützky \& Rainer 2011). For instance, there may be a rule (based on either metaphor or metonymy) that a derived noun with agentive meaning can also be used with instrumental meaning, but this does not relieve the linguist from the task of specifying for each individual lexical item whether it has agentive meaning, instrumental meaning, or both. Moreover, an existing derived noun may have only the instrumental meaning. Hence, as argued in Booij (2010), we need to specify both meaning contributions of the relevant affixes, whereas the common formal and semantic properties of the various types of derived nouns in -er can be specified in the dominating schema ( $x$ is a variable for 'entity' which receives a more precise semantic specification in the subschemas):

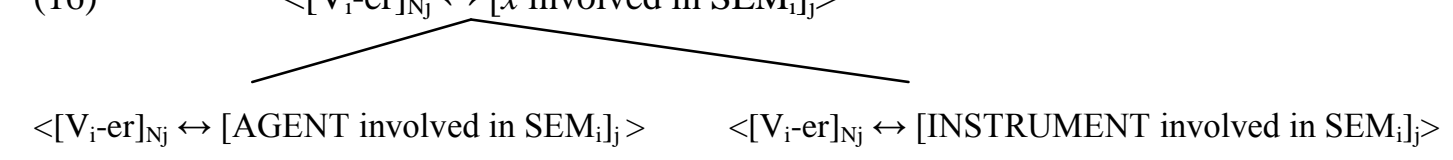

(Other possible meanings of these -er nouns such as place (diner) are not dealt with here, but will have to be added on the bottom line of (16)).

Each subschema heads the individual agent and instrument nouns. If a noun in -er is polysemous (for instance, the Dutch noun zender 'sender', which can be interpreted as either an agent or an instrument), this word will have two subentries, one for the agent interpretation and one for the instrument interpretation. Hence, the noun zender will be specified as one form correlating with two meanings and will be linked to both subschemas.

$$
\left.<\left[[z e n d]_{\mathrm{V}_{\mathrm{i}}}-\mathrm{er}\right]_{\mathrm{Nj}} \leftrightarrow\left\{<\left[\text { AGENT involved in } \mathrm{SEM}_{\mathrm{i}}\right]_{\mathrm{j}}>, \text { [INSTRUMENT involved in } \mathrm{SEM}_{\mathrm{i}}\right]_{\mathrm{j}}>\right\}
$$

The advantage of this representation of polysemy is that it does not presuppose that the grammar of the individual languages involved contains a derivational rule of sense extension that has to derive the instrumental meaning for each individual word from an agentive meaning. This is the more so important because the polysemy of this type of nouns may not be due to a (metaphorical or metonymic) mechanism of sense extension but to other causes such as the conflation of historically different suffixes, or ellipsis in phrases with a head noun denoting the instrument, e.g. French appareil régulateur > regulateur 'regulating mechanism' (Rainer 2011). In addition, this representation does not claim that each individual instrumental noun in -er is derived from the same noun with agentive meaning. There are many instrumental nouns in Dutch 
that lack an agentive counterpart, although the word with the agentive meaning is potentially well-formed.

In sum, there are (possibly universal) schemas of conceptual extension, such as the schema that instruments can be conceived of as impersonal agents (metaphorical extension) or that the notion of agent can be linked to the instrument or the place of action (the mechanism of metonymy), but the actual polysemous words of a language, which may be partially due to such sense extension schemas, need to be specified in the lexicon (Booij 2010). Both word formation schemas and sense extension schemas of the type illustrated here have a motivating function with respect to the existing set of words of a language, and each of them reduces the degree of arbitrariness between form and meaning in its own way.

\section{Affixoids and polysemy}

Words may have special meanings when embedded in complex words. For example, the Dutch noun hoofd 'head' carries the meaning 'main' when embedded in a compound. Hence, the basic meaning of hoofd, namely 'head', can be overridden in compounds like hoofd-bezwaar 'main objection'. The meaning of a constituent word can be overridden when embedded in a complex word, as we saw in section 5, illustrated by the word wonder-lijk 'strange' in which the meaning of the base word wonder 'miracle' has become opaque. That is, semantic properties of word constituents can be overridden. The difference in the case of the compound hoofd-bezwaar is that the interpretation of hoofd as 'main' is not just present in this compound alone, but is a systematic option for speakers of Dutch, as illustrated by the following examples:

$$
\begin{aligned}
& \text { hoofd-bezwaar 'main objection' } \\
& \text { hoofd-gedachte 'main idea' } \\
& \text { hoofd-ingang 'main entrance' }
\end{aligned}
$$

We can describe this type of polysemy by means of a lexical subentry for hoofd with the bound meaning, bound to a specified morphological context. The word hoofd is polysemous, with at least the following meanings:

a. upper part of the body (as in hoofd-pijn 'head-ache')

b. entity at the top of a hierarchy (as in het hoofd van de afdeling 'the head of the department' and hoofd-kantoor 'head office') 
c. (as first part of a compound): main, most important, as in hoofd-bezwaar 'main objection')

The relevant constructional idiom for meaning (c) is

$$
<\left[[\text { hoofd }]_{\mathrm{Ni}} \mathrm{N}_{\mathrm{j}}\right]_{\mathrm{Nk}} \leftrightarrow\left[\mathrm{MAIN}_{\mathrm{i}} \mathrm{SEM}_{\mathrm{j}}\right]_{\mathrm{k}}>
$$

This meaning of the polysemous word hoofd is unique to the compounding configuration, as it does not occur by itself. For instance, one cannot say *Dit problem is het hoofd instead of Dit is het hoofdprobleem 'This is the main problem'. Therefore, the meaning 'main' for hoofd is not a case of context-free sense extension, but is context-dependent.

The case of the affixoid hoofd-is a case of construction-dependent polysemy: this meaning of the noun hoofd is specified as occurring in the left position of NN compounds only. The relationship between this affixoid hoofd-and the other lexical subentries for (the various meanings of) hoofd is one of polysemy.

The specification of construction-dependent interpretations of words in the grammar is in conformity with the observation that this kind of polysemy is conventional and language-specific. Note that the meaning 'main' of hoofd-is not generally available for its English counterpart head: one cannot speak of the head issue if what is meant is the main issue. In German, on the other hand, the dependency on compound structure of the corresponding noun Haupt- has become even stronger, as it is no longer used productively as a free word. Hence, it is now qualified as a prefix in grammars of German.

What we observe here is that words may have bound meanings, that is, meanings tied to specific lexical constructions. The relevance for the issue of default override is that the literal meaning of hoofd appears to be a defeasible semantic property that is overridden in the compounds listed in (18). We specify a subclass of all NN compounds with hoofd as their modifier, as part of the hierarchy for nominal compounds, by means of the constructional idiom (19). This constructional idiom provides motivation for these compounds and makes them transparent, even though the regular literal meaning 'head' of the left constituent hoofd is not present in these compounds. Schema (19) is located at an intermediate level in between the general schema for NN compounds and the individual compounds with hoofd-, and partially overrides the otherwise predictable meaning of these NN compounds with hoofd as their left constituent. Hence, this type of subschema is different from the subschemas in (11) that provide more detailed specifications of properties mentioned in the general schema, but do not override properties predicted by the general schema. 
In conclusion, there are systematic patterns of polysemy in complex words. They can be dealt with in terms of subschemas that partially override the meaning predicted by more general schemas. Thus, we see that deviations from expected properties may be systematic in nature, due to a local generalization, such as the productive meaning 'main' for hoofd as first constituent of an NN compound. For these cases, default override is not a matter of the properties of individual complex words, but of a subschema that override properties predicted by a schema at a higher level in the hierarchical lexicon.

\section{Allomorphy}

The variation in the shape of a morpheme may not be governed by a transparent phonological rule or constraint. Consider, for instance, the following set of related words in Dutch:

$$
\begin{array}{ll}
\text { aanva[y] 'begin' } & \text { aanva[yk]-elijk 'initially' } \\
\text { toega[y] 'access' } & \text { toega[yk]-elijk 'accessible' } \\
\text { afha[y] 'to depend' } & \text { afha[yk]-elijk 'dependent' }
\end{array}
$$

When we compare the phonological forms of the base words when used as independent words and when used as building blocks of the derived adjectives, we observe a phonological difference, as there is an extra stem-final $/ \mathrm{k} /$ in the derived adjectives. No productive phonological rule of Dutch can account for this alternation, and hence this type of allomorphy has to be memorized for these derived adjectives. Yet the relation between the two sets of word is transparent. That is, a certain degree of allomorphy does not impede recognition of lexical relationships. Therefore, we may conclude that complete inheritance of the phonological shape of the base word is not necessary in order to recognize a relationship: in other words, phonological shape is, to a certain extent, a defeasible property, as long as the semantic relationships involved are clear enough. This is also the conclusion drawn for Italian by Celata \& Bertinetto (2005), as discussed in Booij (2010: Chapter 10). Palatalization of consonants before the vowel /i/ in Italian has also become a lexically governed rule, as illustrated by the following examples:

$$
\begin{array}{ll}
\operatorname{ami}[\mathrm{k}]-\mathrm{o}-\operatorname{ami}[\mathrm{tš}]-\mathrm{i} \text { 'friend(s)' } & \operatorname{ami}[\mathrm{tš}]-i z i a \\
\text { mendi[k]-o - mendi[k]-i 'mendicant(s)' } & \text { mendi[tš }] \text {-izia 'mendicity' }
\end{array}
$$


In the first example, palatalization applies across the board, whereas in the second example, the plural form of mendico has no palatalization. These facts show that the effects of phonological processes may have to be encoded in lexical representations.

Psycholinguistic experiments have confirmed that allomorphy in related words does not impede the establishment of paradigmatic relations. For example, German diminutive forms have the same priming effect on the recognition of their base nouns, regardless of whether or not they have a stem vowel change (Clahsen et al. 2003: 140). Stem vowel change in German diminutives (Umlaut) is not automatic, as shown by the following pairs of related words:

Vater 'father' - Väter-chen 'father, diminutive'

Onkel 'uncle' - Onkel-chen 'uncle, diminutive'

In the second pair, the full vowel in the first syllable of the base is not fronted in the diminutive noun. Yet Onkelchen does not differ significantly from Väterchen in its priming effect with respect to its base word.

There is also no effect on priming of Italian stem allomorphy in plural nouns with palatalization, such as amico-amici 'friend(s)', as opposed to those without, such as buco - buchi 'hole(s)' (Celata and Bertinetto 2005). For instance, if the target word is drastic-o, then the words drasti[k]-i and drastic[ťs]-i have the same priming effect.

In their study of the effects of morphological relatedness on word recognition (the family size effect) for Dutch language users, De Jong et al. (2000: 353) concluded that 'the effect of Family Size is not mediated by the exact form of the base word, but by a more abstract central morphological representation'. For instance, there are family size effects for a verb and its irregular participle, as in bind 'bind'-ge-bond-en 'bound' which differ in the phonological form of their stem.

To conclude, allomorphy does not impede the establishment of relations between words, which is a robust process. Finding a relation of motivation between a complex word and its base word(s) is always a matter of shared semantics, and if the relevant words share semantic properties, phonological variation is less of a problem. That is, the relationship between a complex word and its base word(s) is not necessarily obscured by phonological differences. It is not an absolute precondition for establishing lexical relatedness between a complex word and its base(s) that the former should contain constituents in exactly the phonological form seen in the latter. Therefore, complex words may receive motivation from being linked to base words even if they show some phonological dissimilarities. The predicted phonological form of the base word 
part of a complex word is therefore a property that can be overridden by the type of phonological variation that we refer to as allomorphy: forms identical in meaning and similar in phonology. Allomorphy does not necessarily reduce the degree of motivation of a complex word.

\section{Conclusions}

Let us now return to the basic questions addressed in this paper: what is the status of inheritance and of default inheritance in the framework of CM? As there is no underspecification of lexical properties in $\mathrm{CM}$, this question has had to be rephrased in two steps, as: (i) in which ways can the form-meaning relationship of a complex word be motivated? (ii) when will the absence of properties in a complex word that are predicted by a word formation schema lead to complete blocking of the motivation relation between the complex word and the schema in question?

The answers proposed here are the following:

(i) the form-meaning relation of a complex word may be motivated by schemas and subschemas, by its relation with a base word, by its paradigmatic relation to another complex word, and by schemas for regular polysemy.

(ii) a property predicted for a complex word by the relevant schema can be overridden by that word unless the property has to be considered absolute. This is default override. Default override implies that a complex word may lose part of its motivation. The only candidates for absolute, non-defeasible properties in word formation schemas are the output category of complex words and the phonological shape of their constituent morphemes. However, phonological shape can vary within the boundaries of allomorphy.

If there is a systematic and extendable subpattern with an unpredicted, new meaning at stake (as in the case of Dutch compounds with the noun hoofd-), we can make use of subschemas which state the relevant generalizations, and which override properties specified at a higher level in the hierarchical lexicon. Such subschemas (constructional idioms) thus contribute to the (re-)motivation of complex words. Thus, the concept of a hierarchical lexicon, with schemas and subschemas, provides an excellent model for dealing with such facts.

A lexicon with full entries avoids the problem of how to formalize default override, in particular in the domain of semantic properties which cannot obviously be specified in terms of feature: value combinations. Instead, complex words are supposed to differ in their degree of motivation. The degree of motivation is inversely proportional to the number of properties overridden at lower levels. However, schemas for polysemy and constructional idioms serve to introduce motivation on lower levels, and hence increase the degree of motivation. 
Being a motivated (non-arbitrary) linguistic sign is a gradient property of complex words that correlates with the degree to which the formal and semantic properties of the relevant word formation schema and of its base word(s) have been preserved.

*The research for this paper has been supported by a Humboldt Research Award from the Alexander von Humboldt-Stiftung, Bonn, which is gratefully acknowledged here. I would like to thank Jenny Audring (Leiden), Matthias Hüning (FU Berlin), Steven Kaye (Oxford), and Ton van der Wouden (Meertens-Instituut Amsterdam) for their constructive remarks on an earlier draft of this text.

Note

1. The use of default inheritance can be seen as logically independent of the idea of impoverished lexical entries, as the following quotation from Bouma (1993: 49) makes clear:

\begin{abstract}
"An important constraint on the use of default unification is that I will assume that it is only used to streamline grammatical descriptions. That is, default unification is used to implement non-monotonic template inheritance and lexical feature specification defaults in the context of unification grammar. The effects of these extensions can be computed at 'compile-time', however, that is, during the process of compiling a grammar definition into a set of grammar rules and lexical items associated with certain feature structures. Grammatical processing (i.e. parsing or generation) only needs to operate on the latter kind of objects, and thus, is completely unaffected by the introduction of non-monotonic devices for defining grammatical objects. The advantage of this point of view is that it enables us to incorporate a number of powerful non-monotonic description methods, while at the same time, the grammar can still be considered as a declarative characterization of the set of well-formed derivation trees." (Bouma 1993: 49).
\end{abstract}

That is, there is no clash between a full entry theory and the use of default inheritance as a descriptive device.

References 
Baayen, R. Harald, James M. McQueen, Ton Dijkstra \& Rob Schreuder. 2003. Frequency effects in regular inflectional morphology: Revisiting Dutch plurals.

Morphological structure in language processing, ed. by R.H. Baayen \& R.

Schreuder, 355-90. Berlin: Mouton d Gruyter.

Blank, Andreas. 2003. Polysemy in the lexicon and in discourse. Polysemy. Flexible patterns of meaning in mind and in language, ed. by B. Nerlich, Z. Todd, V.

Herman \& D.D. Clarke, 267-96. Berlin / New York: Mouton de Gruyter.

Bloomfield, Leonard. 1935. Language. London: Allen and Unwin.

Booij, Geert. 2010. Construction morphology. Oxford: Oxford University Press.

Bouma, Gosse. 1993. Nonmonotonicity and Categorial Unification Grammar. Groningen: University of Groningen.

Bybee, Joan. 2006. From usage to grammar: the mind's response to repetition. Language 82.711-33.

Celata, Chiara \& Pier Marco Bertinetto. 2005. Lexical access in Italian: words with and without palatalization. Lingue e linguaggio 2.293-318.

Clahsen, Harald, Ingrid Sonnenstuhl \& James P. Blevins. 2003. Derivational morphology in the German mental lexicon: A dual mechanism account. Morphological structure in language processing, ed. by R.H. Baayen \& R. Schreuder, 125-56. Berlin: Mouton de Gruyter.

Corbett, Greville G. \& Norman M. Fraser. 1993. Network Morphology, a DATR account. Journal of Linguistics 29.113-42.

De Vaan, Laura, Rob Schreuder \& R. Harald Baayen. 2007. Regular morphologically complex neologisms have detectable traces in the mental lexicon. The Mental Lexicon 2.1-32

Deo, Ashwini. 2007. Derivational morphology in inheritance-based lexica: Insights from Panini. Lingua 117.175-201.

Flickinger, Daniel Paul. 1987. Lexical rules in the hierarchical lexicon. Stanford: Stanford University dissertation.

Goldberg, Adele. 2006. Constructions at work. The nature of generalization in language. Oxford: Oxford University Press.

Hay, Jennifer \& R. Harald Baayen. 2005. Shifting paradigms: Gradient structure in morphology. Trends in Cognitive Sciences 9.342-48.

Hudson, Richard. 2007. Language networks. The new word grammar Oxford: Oxford University Press.

Jackendoff, Ray. 1975. Semantic and morphological regularities in the lexicon. Language 51.639-71.

Jackendoff, Ray. 1997. The architecture of the language faculty. Cambridge Mass.: MIT Press.

Jackendoff, Ray. 2002. Foundations of language. Oxford: Oxford University Press.

Jackendoff, Ray. 2011. What is the human language faculty? Two views. Language 87.586-624.

Kilbury, James, Wiebke Petersen \& Christoph Rumpf. 2006. Inheritance-based models of the lexicon. Advances in the theory of the lexicon, ed. by D. Wunderlich, 429-80. Berlin: Mouton de Gruyter.

Luger, G. \& W. Stubblefield. 1993. Artificial intelligence: Structures and strategies for complex problem solving. New York: Benjamin Cummings. 
Luschützky, Hans \& Franz Rainer. 2011. Introduction. Agent noun polysemy in IndoEuropean languages. Language Typology and Universals 64.1-7.

Müller, Stefan. 2007. Head-Driven Phrase Structure Grammar: Eine Einführung. Tübingen: Stauffenburg Verlag.

Pinker, Steven. 1999. Words and rules. New York: Basic Books.

Plag, Ingo. 2004. Syntactic category information and the semantics of derivational morphological rules. Folia Linguistica 38.193-225.

Rainer, Franz. 2011. The agent-instrument-place "polysemy" of the suffix -TOR in Romance. Language Typology and Universals 64.8-32.

Riehemann, Suzanne Z. 1998. Type-based derivational morphology. Journal of Comparative Germanic Linguistics 2.49-77.

Sag, Ivan A., Thomas Wasow \& Emily M. Bender. 2003. Syntactic theory. A formal introduction. Stanford, Calif.: CSLI Publications.

Smedts, Willy. 1979. Lexicale morfologie. De beheersing van de woordvorming door Vlaamse 'brugklassers'. Leuven: Katholieke Universiteit Leuven.

Tomasello, Michael. 2003. Constructing a language: A usage-based account of language acquisition. Cambridge MA: Harvard University Press.

Zeschel, Arne. 2009. What's (in) a construction? Complete inheritance vs. full-entry models. New directions in Cognitive Linguistics, ed. by V. Evans \& S. Pourcel, 185-200. Amsterdam / Philadelphia: Benjamins. 